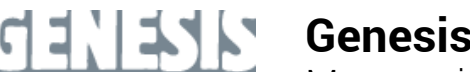

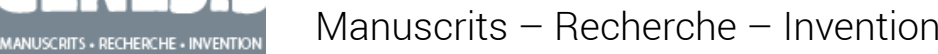

33 | 2011

Afrique-Caraïbe

\section{Nouvelles d'archives}

Lydie Rauzier

\section{OpenEdition}

Journals

Édition électronique

URL : http://journals.openedition.org/genesis/672

DOI : 10.4000/genesis.672

ISSN : 2268-1590

\section{Éditeur :}

Presses universitaires de Paris Sorbonne (PUPS), Société internationale de génétique artistique littéraire et scientifique (SIGALES)

\section{Édition imprimée}

Date de publication : 30 octobre 2011

Pagination : 197-201

ISBN : 978-2-84050-804-5

ISSN : 1167-5101

\section{Référence électronique}

Lydie Rauzier, « Nouvelles d'archives », Genesis [En ligne], 33 | 2011, mis en ligne le 23 octobre 2013, consulté le 15 septembre 2020. URL : http://journals.openedition.org/genesis/672 


\title{
Nouvelles d'archives
}

\author{
Lydie Rauzier*
}

Pour ce numéro, la rubrique "Nouvelles d'archives » recense, pour l' année 2010, les enrichissements de la Bibliothèque nationale de France (département des Manuscrits et département des Arts du spectacle), ceux de la Bibliothèque littéraire Jacques Doucet et ceux de l'Institut mémoire de l'Édition contemporaine (IMEC).

Les documents qui viennent enrichir un fonds déjà constitué sont introduits par un astérisque et les fonds nouvellement constitués sont indiqués en caractères gras.

\section{Aвout Edmond}

Correspondance (dont lettres de Benjamin Constant, Coquelin aîné, Vidocq) ; manuscrits ; volumes de l'œuvre, documents présentés lors d'une exposition : correspondance de Gustave Flaubert, Ivan Tourgueniev, Eugène Labiche, Léon Gambetta, Adolphe Thiers, Jules Ferry.

Institut Mémoires de l'Édition contemporaine

\section{Adonis}

Outre une riche bibliothèque d'études comportant ses ouvrages en éditions originales et leurs traductions dans plus de vingt-cinq langues, le fonds comporte les dossiers des nombreuses et violentes polémiques suscitées par les écrits d'Adonis et ses prises de position politiques et culturelles. S'y ajoutent enfin les archives des deux revues mythiques qui ont joué un rôle prépondérant dans le renouveau intellectuel arabe : Ch'ir, fondée en 1957 avec Yussuf El Khâl, et Mawâqif, créée en 1968 et qu'il dirigea durant trente ans. Le fonds Adonis rejoint à l'IMEC ceux de nombreux auteurs issus du monde arabe, Andrée Chedid, Georges Schehadé ou Kateb Yacine.

Institut Mémoires de l'Édition contemporaine

\section{Andreu Pierre}

Manuscrits, dossiers de travail - notamment ceux constitués pour ses recherches biographiques - et carnets de captivité alors qu'il était au stalag. Une riche correspondance intellectuelle avec Pierre Drieu La Rochelle, Max Jacob, Charles Maurras, Pierre Mac Orlan, Henry de Montherlant, Jacques Maret (directeur de la revue Les Feuillets inutiles) et une importante collection de revues des années trente complètent cet ensemble.

Institut Mémoires de l'Édition contemporaine

BALTAZAR Julius

Ensemble de correspondance reçue. Certaines lettres ont été enluminées par Julius Baltazar après réception.

Chancellerie des Universités de Paris, Bibliothèque littéraire Jacques Doucet

\section{BARTHES Roland}

Une vie d'écriture à travers les manuscrits de l'essentiel de ses œuvres (et de manière exhaustive à partir de 1970, c'est-à-dire de $S / Z$ à La Chambre claire), ses articles, conférences, entretiens, mais aussi ses cours et séminaires (tant à l'École Pratique des Hautes Études qu'au Collège de France), ainsi que des carnets, d'immenses fichiers de travail, les correspondances reçues à l'occasion de la parution de ses livres, et un vaste ensemble de dessins.

Bibliothèque nationale de France, département des Manuscrits

\section{BENÔ̂T Jean}

Ensemble de textes relatifs à ses conquêtes amoureuses, mis bout à bout et conservés sous forme de rouleaux. Un rouleau est constitué par les enveloppes décorées envoyées par Marie Morel à Jean Benoît. Certaines sont décorées de plumes.

Chancellerie des Universités de Paris, Bibliothèque littéraire Jacques Doucet

\footnotetext{
*Avec l'aide d'Hélène Favard (IMEC), Marie Odile Germain (BnF, département des Manuscrits), Joël Huthwohl (BnF, département des Arts du spectacle), Marie-Dominique Nobecourt Mutarelli (Bibliothèque littéraire Jacques Doucet).
} 
BiLly André

Poèmes, pièces de théâtre, récits.

Bibliothèque nationale de France, département des Manuscrits

\section{BRESNER Lisa}

Manuscrits, carnets de notes souvent ornés de dessins, importante documentation sur la Chine traditionnelle et moderne (culture, calligraphie, art, littérature populaire, dictionnaires), enregistrements audio et vidéo, documents biographiques, photographies, lettres reçues, dossiers de presse liés à ses publications et quelques livres de sa bibliothèque personnelle.

Institut Mémoires de l'Édition contemporaine

Butor Michel

Lettres autographes signées à Julius Baltazar.

Chancellerie des Universités de Paris, Bibliothèque littéraire Jacques Doucet

CARADEC François

Correspondance autographe et documents adressés à Anatole Jakovsky, 80 lettres autographes signées, 39 cartes postales autographes signées dont certaines avec collages originaux, 13 billets autographes signés, 30 lettres dactylographiées signées, 3 télégrammes, 2 collages sur carton, enveloppes dont certaines avec collages ou dessins (19541976)

Chancellerie des Universités de Paris, Bibliothèque littéraire Jacques Doucet

\section{Casanova Giacomo}

Plus de 3700 pages d'une écriture serrée, émaillée de ratures, surcharges et noms biffés : un ensemble exceptionnel de manuscrits autographes parmi lesquels la légendaire Histoire de ma vie, commencée en 1789 , et maintes fois reprise. La destinée du manuscrit enfin parvenu à la $\mathrm{BnF}$ aura été presque aussi aventureuse que la vie de son auteur.

Bibliothèque nationale de France, département des Manuscrits

\section{Castoriadis Cornelius}

Manuscrits, notes et dossiers de travail, correspondance, dossiers professionnels, dossiers concernant Socialisme ou Barbarie ainsi que divers groupes de la gauche radicale. Séminaires, photographies, enregistrements audios et vidéos, travaux universitaires, bibliothèque d'étude.

Institut Mémoires de l'Édition contemporaine

*Chaillou Michel

Deux nouveaux ajouts au fonds Michel Chaillou : Le Dernier des Romains (2009), Le Crime du beau temps (2010).

Bibliothèque nationale de France, département des Manuscrits

*[Char René, Knutson Greta]

Documents relatifs à la participation à la Résistance de Greta Knutson et René Char, certificats dactylographiés et imprimés avec mentions manuscrites dont 2 photocopies, 1 coupure de presse, 1 photo.

Chancellerie des Universités de Paris, Bibliothèque littéraire Jacques Doucet

*Cixous Hélène

Manuscrits de travail de ses derniers textes, pour le théâtre : Les Naufragés du Fol Espoir (2010), mis en scène par Ariane
Mnouchkine au Théâtre du Soleil (Prix Molière 2010 du théâtre public), et la « fiction »: Double Oubli de l'Orang-Outang (2010).

Bibliothèque nationale de France, département des Manuscrits

Cocteau Jean

Lettre autographe signée à François Mauriac, vers 1913.

Chancellerie des Universités de Paris, Bibliothèque littéraire Jacques Doucet

\section{Collection André Vasseur, Fondation Robert Ardouvin}

Environ 750 titres de revues littéraires et artistiques des XIXe et $\mathrm{XX}^{\mathrm{e}}$ siècles. La plupart des titres sont enrichis de photographies, dessins, correspondances et accompagnés de précieuses notes bibliographiques.

Institut Mémoires de l'Édition contemporaine

Comédie-FrançAISE

39 costumes provenant de la Comédie-Française (1857-1992).

Bibliothèque nationale de France, département des Arts du spectacle

CoppéE François

Poèmes manuscrits, 1 cahier.

Bibliothèque nationale de France, département des Manuscrits

\section{DEBORD Guy}

Classées trésor national en 2009, les archives de Guy Debord ont rejoint les collections du département des Manuscrits, une première partie en 2010, la seconde en 2011. Ce fonds comprend toutes les versions de ses écrits et films, une importante correspondance, ses papiers personnels, dossiers de presse, dossiers éditoriaux, carnets et fiches de lectures, notes préparatoires à ses œuvres cinématographiques, archives photographiques, objets personnels, sa bibliothèque, mais aussi des documents de travail de l'Internationale situationniste et des publications de divers groupes d'avant-garde.

Bibliothèque nationale de France, département des Manuscrits

\section{Decourcelle Pierre}

Correspondance littéraire (Paul Bourget, Louis Barthoux, Tristan Bernard, Henri Becque, Aristide Bruant, Henry Bernstein, Alphonse Daudet, Maurice Donnay, Alfred Dreyfus, Henri Duvernois, René Doumic, Arthur Conan Doyle, Firmin Gémier, Lucien et Sacha Guitry, Yvette Guilbert, Ricado Estrada, G. Toudouze, Aurélien Scholl, etc.) bibliothèque d'études et catalogues de ventes de ses collections d'art. Institut Mémoires de l'Édition contemporaine

\section{DELAY Florence}

Archives de ses activités dans le domaine du théâtre et du cinéma en tant qu'auteur, traducteur et comédienne : manuscrits, notes, documentation, photographies.

Bibliothèque nationale de France, département des Arts du spectacle

\section{DELAY Florence}

Ensemble de manuscrits.

Chancellerie des Universités de Paris, Bibliothèque littéraire Jacques Doucet

\section{*Delay Jean}

Ensemble de correspondance reçue.

Chancellerie des Universités de Paris, Bibliothèque littéraire Jacques Doucet 
*DESNOS Robert

1 lettre autographe signée au président du Cercle de la librairie, recommandation de Daniel Dreuil, 2 p. sur 1 feuillet à en-tête d' " Aujourd'hui " (6 décembre 1942) ; 1 lettre autographe signée à Daniel Dreuil. Joint feuillet avec envoi de Desnos à Daniel Dreuil et 1 carte de visite de Desnos, 2 p. sur 1 feuillet à en-tête d' « Aujourd'hui » (25 novembre 1942) ; 10 lettres autographes signées et 2 notes de la générale Moslard, à Daniel Dreuil, relatives à l'arrestation et à la déportation de Robert Desnos (vers octobre 1945).

Chancellerie des Universités de Paris, Bibliothèque littéraire Jacques Doucet

\section{Du Bouchet André}

Ensemble de manuscrits, correspondance, imprimés et documents, dont un ensemble relatif à ses traductions de Paul Celan, Emily Dickinson, Boris Pasternak, aux traductions de ses œuvres par Paul Celan (1946-1997).

Chancellerie des Universités de Paris, Bibliothèque littéraire Jacques Doucet

\section{DuPuy Françoise et Dominique}

Archives administratives, presse, programmes, enregistrements sonores, films, photographies, affiches et maquettes de décors et costumes, qui concernent leur compagnie, Les Ballets modernes de Paris (1955-1978), le Festival des Baux de Provence (1962-1969) et les Journées de la danse.

Bibliothèque nationale de France, département des Arts du spectacle

\section{ÉDitions Minard/LetTres MODERnES}

Bibliothèque de référence.

Institut Mémoires de l'Édition contemporaine

\section{ÉDITIONS DE L'ORANTE}

Dossiers éditoriaux, notamment autour de l'édition de l'Histoire des conciles et de l'édition des œuvres complètes de Søren Kierkegaard. Dossiers se rapportant au rôle joué par Jacques Lafarge au sein du Syndicat national de l'édition ; dossiers de presse ; bibliothèque de référence.

Institut Mémoires de l’Édition contemporaine

*Fardoulis-Lagrange Michel

33 lettres autographes signées à Bernard Guillemot (1983-1988).

Chancellerie des Universités de Paris, Bibliothèque littéraire Jacques Doucet

*Fernandez Dominique

Pise 1951 (paru en 2011) et autres textes récents, manuscrits et sorties de traitement de texte corrigées.

Bibliothèque nationale de France, département des Manuscrits

*Fondane Benjamin

Manuscrits autographes signés, formats divers (1912-1920) : Recenzii III. Mihail Eminescu. Poezii (1912-1914), Eminescu si cosbuc. Claudia Milian (1918-1920) ; Adrian Maniu. Iasi, 1917 ; Fabula lui Iorga (la fable de Iorga) (1918-1920) ; Vlahuta dreptate (vlahita justice) (1914) ; manuscrits autographes signés pour la plupart inédits : Precuvintare pentru ziarul nostru (préface pour notre journal) (1913) ; textes en français (circa 1918), In ultimea enorma (dans la foule énorme) (circa 1917) ; Clavirul (le piano) (1915); Singuratatea (la solitude) (circa 1915) ; Ulisse (Ulysse), poème autographe signé (Jassy, 17-18 septembre 1914) ; Nu vezi cum lacul (Tu ne vois pas comme le lac), poème autographe (1914) ; poème autographe en roumain, variante très différente du poème Sonet. Privelisti (Paysages), poème autographe (1917) ; Psalmul lui adam, poème autographe (circa 1917) ; Biografie, poème autographe signé (Jassy, 1917. 1914-1917) ; manuscrits autographes signés en grande partie inédits : Orasul meu (ma ville) (circa 1915); A doua capitala (la deuxième capitale) (1915) ; Herta (circa 1915) ; Herta, poèma in proza (circa 1915) ; Monologul nasului (le monologue du nez), manuscrit autographe signé (circa 1918), projet d'une pièce de théâtre ; manuscrits autographes signés (1919-1921) : Burghezia noastra (notre bourgeoisie) (1920-1921) ; Loreley (circa 1919) ; manuscrits autographes signés (1916) : Cum m'am nascut (comme je suis né) (1916) ; [Pagini dintr' un confesional (pages d'un confessionnal)] (1916) ; carnet original, avec une poésie autographe, datée 19 mai 1914, signée Beno et dédiée à sa sœur Rodica. Illustré de 11 dessins originaux de Ross, 19 mai 1914 ; Dorm florile (les fleurs dormant), pastel ; Idilà, poèmes autographes, 2-12 avril 1914 ; carnet original, avec un dessin en frontispice. Notes, titres de ses poésies en français et en roumain (vers 1922) ; lettres autographes signées : avec Luca Gridu ; deux lettres à Rodica (circa 1923 et Paris, 1925). Avec Lina Pascal-Fondane, carte postale autographe signée à Rodica et à Adela (Paris, 1931). Lettre tapuscrite à Rodica (Paris, 1932). Lettre à Rodica (Paris, circa 1935) ; lettre autographe signée à Raymond Queneau (juin 1939).

Chancellerie des Universités de Paris, Bibliothèque littéraire Jacques Doucet

\section{Fourcade Dominique}

Manuscrits autographes ou dactylographiés, dont ceux de Rose-déclic, Le Ciel pas d'angle, Son blanc du un, Élégie L apostrophe E.C., Une histoire ininterrompue, Outrance utterance, Au travail ma chérie, IL, Ensemble, Oh mais qu'est-ce que, Compact pour Claude, Degas parti se changer, Citizen, L'Hymne désormais vain, Contrat ordinaire, etc. ; 881 lettres adressées à Gérard Masson.

Chancellerie des Universités de Paris, Bibliothèque littéraire Jacques Doucet

*GENEFORT Laurent

Carnets de notes préparatoires pour un roman de science-fiction.

Bibliothèque nationale de France, département des Manuscrits

*Giraudoux Jean

Églantine, épreuves corrigées.

Bibliothèque nationale de France, département des Manuscrits

*Guyotat Pierre

(Euvres en cours (« Labyrinthe », « Carnets de bord »), fichiers électroniques sur clé USB dont il a été tirée une sortie papier.

Bibliothèque nationale de France, département des Manuscrits

\section{Houdart Dominique et Heuclin Jeanne}

Archives de la compagnie Houdart-Heuclin contenant des documents audiovisuels, des affiches, des documents papier, 22 marionnettes, 4 masques et 15 accessoires.

Bibliothèque nationale de France, département des Arts du spectacle 
*Jeannet Frédéric-Yves

Trois états dactylographiques corrigés de son dernier texte, Osselets, paru en 2010.

Bibliothèque nationale de France, département des Manuscrits

\section{KaLISKy René}

Manuscrits de l'œuvre théâtrale, notes et dossiers de travail, dossiers de presse, iconographie, bibliothèque de travail.

Institut Mémoires de l'Édition contemporaine

\section{Lacoue-Labarthe Philippe}

Le fonds restitue la richesse des thèmes de recherche du philosophe. L'ensemble inédit le plus conséquent est constitué par les textes ayant servi de support aux cours et séminaires de Philippe Lacoue-Labarthe : de là, on peut suivre le mouvement qui transformait certains séminaires en texte de conférence, certaines conférences devenir des articles, la plupart des livres étant ensuite composés d'articles rassemblés ou redéveloppés.

Institut Mémoires de l'Édition contemporaine

*Léautaud Paul

1 dossier : Paul Léautaud, Petit débat littéraire, constitué de 3 lettres autographes signées de Paul Léautaud à François Caradec (15, 18 et 20 octobre 1948), sur divers supports, 2 enveloppes, 2 feuillets d'épreuves corrigées du texte de Léautaud Petit débat littéraire avec commentaires de l'auteur, 1 exemplaire de la plaquette imprimée en 1948, 1 exemplaire de la revue Caractère de mars 1972 dans laquelle François Caradec a publié les lettres et les épreuves, sous le titre « À un jeune imprimeur : trois lettres inédites de Léautaud » (1948-1972). Chancellerie des Universités de Paris, Bibliothèque littéraire Jacques Doucet

\section{L'Herbier Marcel}

Archives de sa carrière de cinéaste et de producteur. Scénarios, dossiers de productions, photographies, presse, correspondance, dossiers sur l'IDHEC, sur l'Association des auteurs de films, manuscrits littéraires, porte-voix.

Bibliothèque nationale de France, département des Arts du spectacle

*Malraux André

Ensemble de manuscrits d'André Malraux : Lazare, La Métamorphose des dieux et un manuscrit inédit, Le Maquis, suite du dépôt du legs André Malraux.

Chancellerie des Universités de Paris, Bibliothèque littéraire Jacques Doucet

\section{*Memmi Albert}

Manuscrits de travail (Éloge des femmes, Testament insolent) et agendas. Bibliothèque nationale de France, département des Manuscrits

\section{Mrzón Luis}

Différents textes manuscrits : Cinq poemas del mar de las Sargazes ; Le Secret de Baltazar, incomplet ; Musculatura erotica; Soy una naranja; La Marajada; La Memoria del agua; Poèmes d'eau et de lumière, tapuscrit et épreuves corrigées.

Chancellerie des Universités de Paris, Bibliothèque littéraire Jacques Doucet
Nouveau Germain

Carnet de notes avec dessins, manuscrit autographe.

Chancellerie des Universités de Paris, Bibliothèque littéraire Jacques Doucet

Paulhan Jean

110 lettres autographes et dactylographiées signées, billets et cartes à Jacques Debû-Bridel (1940-1950).

Chancellerie des Universités de Paris, Bibliothèque littéraire Jacques Doucet

*PIAF Édith

Archives sur sa carrière réunie par l'Association des amis d'Édith Piaf : affiches, tableaux (4), documentation, marionnette (1).

Bibliothèque nationale de France, département des Arts du spectacle

\section{*PINGET Robert}

Notre ami Sam, manuscrit dactylographié original et 12 pages manuscrites autographes.

Chancellerie des Universités de Paris, Bibliothèque littéraire Jacques Doucet

\section{*Planchon Roger}

Archives de la Compagnie Roger Planchon, notes de mises en scène pour des spectacles montés ou non montés, concernant les trois périodes (Théâtre de la Comédie, Théâtre de la Cité et TNP).

Bibliothèque nationale de France, département des Arts du spectacle

*Ponge Francis

Ensemble de manuscrits divers : Cahier de notes pour Malherbe ; « Fanfare pour Jean Hélion. Pour le catalogue de son exposition CNAC au GrandPalais. Octobre-novembre $1970 » ;$ Le Pré (Skira), manuscrits autographes, manuscrits dactylographiés (1960-1970), dont manuscrits inédits ; correspondance éditoriale avec Gaëtan Picon au sujet du Pré; dossier de réception du Pré ; dossier de presse ; manuscrits divers sur Fautrier ; La Nioque, manuscrit autographe, manuscrit dactylographié, épreuves corrigées ; dossier de correspondance éditoriale ; «Préface à un bestiaire »; « Qualité de Paul Éluard »; « Jean Hytier » et lettres reçues de Jean Hytier ; dossier relatif à sa participation au surréalisme ; texte et documentation à propos de Paul Valéry ; Le Verre d'eau ; fabrique du texte sur Picasso, pour Picasso de Draeger, et correspondance à ce sujet, 1974; 1 carnet autographe ; Maurice Blanchot, lettres autographes reçues de Marie Dormoy, François Chapon, Maurice Blanchot ; Le Carnet du bois de pin, manuscrit autographe.

Chancellerie des Universités de Paris, Bibliothèque littéraire Jacques Doucet

RaCINE Bruno

Manuscrit du Gouverneur de Morée (1982).

Bibliothèque nationale de France, département des Manuscrits

*RÉmY Pierre-Jean

Euvres récentes, articles, conférences.

Bibliothèque nationale de France, département des Manuscrits

*REverdy Pierre

Le Voleur de Talan, manuscrit autographe [1917] ; fragments autographes correspondant à une version primitive du Voleur de Talan et comportant de nombreuses ratures et corrections, 5 p. (1917).

Chancellerie des Universités de Paris, Bibliothèque littéraire Jacques Doucet 
*RoDANSKI Stanislas

Morts avec vous, manuscrit autographe, 1 cahier.

Chancellerie des Universités de Paris, Bibliothèque littéraire Jacques Doucet

\section{ROHMER Éric}

Manuscrits de l'œuvre ; archives consacrées aux films (Perceval, L'Amie de mon amie, Ma nuit chez Maud, La Collectionneuse. L'Anglaise et le Duc, L'Astrée...) ; notes de travail ; dossiers de presse ; archives professionnelles (en tant qu'enseignant, universitaire, critique de cinéma) ; nombreux écrits publiés principalement dans les Cahiers du cinéma et Arts ; correspondances (avec François Truffaut, Paul Gégauff, Alexandre Astruc, Jean Cocteau, Roger Leenhardt, Jacques Doniol-Valcroze, Henri Langlois, Georges Sadoul, Jacques Siclier, Jean Douchet, Serge Daney, Serge Toubiana, Henry Chapier, et de nombreuses lettres d'admirateurs ou de spectateurs) ; iconographie ; archives biographiques.

Institut Mémoires de l'Édition contemporaine

*Rosset Clément

Nouveau complément du fonds manuscrit : Tropiques (2010).

Bibliothèque nationale de France, département des Manuscrits

RousSEL Raymond

Album photographique de Raymond Roussel et de la famille de sa sœur, Germaine Roussel, duchesse d'Elchingen-Ney, 125 photographies. Joints 45 contretypes de photographies anciennes comprenant pour chacune un négatif et un tirage sur papier.

Chancellerie des Universités de Paris, Bibliothèque littéraire Jacques Doucet

\section{Roy Jean}

Correspondance, livres, coupures de presse, formats divers.

Chancellerie des Universités de Paris, Bibliothèque littéraire Jacques Doucet

\section{SCHIFFRIN Jacques}

Ensemble de papiers personnels de Jacques Schiffrin, avec des manuscrits d'autres auteurs et des lettres reçues d'André Gide, Roger Martin du Gard, Georges Duhamel, André Malraux, Marcel Aymé, Julien Green, Jean Bruller, André Maurois, Paul Valéry, etc.

Chancellerie des Universités de Paris, Bibliothèque littéraire Jacques Doucet

*SCHLUMbERGER Jean

Correspondance autographe entre Jean Schlumberger et sa femme, Suzanne Weier Schlumberger (1895-1924), photocopies ; 50 lettres de
Jean Schlumberger à Anne Heurgon, 2 lettres d'Anne Heurgon à Jean Schlumberger, formats divers.

Chancellerie des Universités de Paris, Bibliothèque littéraire Jacques Doucet

*Tellerman Esther

Euvres (suite) : manuscrits, dactylographies corrigées et carnets.

Bibliothèque nationale de France, département des Manuscrits

ThéÂtre de la Huchette

Pour Le Cirque (1982) : maquette en volume de Jacques Noël et photographies de Dominique Rigoleur, entre autres ; sont joints des documents sur les spectacles dans lesquels a joué Jacques Jeannet; don complémentaire de 2 chaises-décor et 4 panneaux de décor signés Erté, de 16 costumes, ainsi que 2 cartons d'archives.

Bibliothèque nationale de France, département des Arts du spectacle

*THÉÂtre DU SOLEIL

18 cartons d'archives sur les spectacles du Théâtre du Soleil, de Gengis Khan à Et soudain des nuits d'éveil ; films, enregistrements sonores et vidéo, rushes du film $\mathrm{Au}$ Soleil même la nuit, entretiens avec Ariane Mnouchkine avec les grandes figures de la Résistance ; une poupéedécor pour le spectacle Sihanouk.

Bibliothèque nationale de France, département des Arts du spectacle

*VALÉRY Paul

Ensemble d'archives intimes provenant de la succession d'Agathe Rouart-Valéry, fille du poète : une trentaine de carnets de notes, de dessins et d'aquarelles, une série d'agendas, plusieurs dossiers manuscrits, des épreuves, des textes de jeunesse, et de nombreuses correspondances, tant familiales (à son frère Jules) que littéraires (lettres de et à Paul Valéry).

Bibliothèque nationale de France, département des Manuscrits

*VIAN Boris

4 nouveaux manuscrits : Conte de fées à l'usage des moyennes personnes, Elles se rendent pas compte, manuscrits autographes ; L'Équarrissage pour tous, manuscrit autographe et dactylographie corrigée ; Et on tuera tous les affreux, articles corrigés.

Bibliothèque nationale de France, département des Manuscrits

*VILMORIN Louise de

Lettres, cartes postales et poèmes à Maurice Pianzola, conservateur du Musée d'Art et d'Histoire de Genève (1961-1967).

Chancellerie des Universités de Paris, Bibliothèque littéraire Jacques Doucet 